\title{
Diagnostic Accuracy of Lateral Decubitus Chest Radiography before Pleural Maneuvers for the Management of Pleurisies in the Era of Chest Ultrasound
}

\author{
Khady Thiam ${ }^{a, b}$ Julie Berbis ${ }^{c}$ Sophie Laroumagne ${ }^{a, d} \quad$ Julien Guinde ${ }^{a}$ \\ Bertrand Chollet $^{a}$ Hervé Dutau ${ }^{a}$ Nafissatou O. Touréb Philippe Astoul ${ }^{a}$ d, e \\ a Department of Thoracic Oncology, Pleural Diseases, and Interventional Pulmonology, Hôpital Nord, Marseille, \\ France; ${ }^{b}$ Department of Pulmonology and Phthisiology, CHNU Fann, Dakar, Senegal; 'Aix-Marseille University, \\ EA 3279, Public Health, Chronic Diseases and Quality of Life, Research Unit, Marseille, France; ${ }^{\mathrm{d}} \mathrm{G}-$ Echo, French \\ National Group for Chest Ultrasonography, Paris, France; ${ }^{\text {e}}$ Aix-Marseille University, Marseille, France
}

\section{Keywords}

Malignant pleural effusions · Chest radiography · Medical thoracoscopy $\cdot$ Pleural adhesion · Chest ultrasound

\begin{abstract}
Background: Chest ultrasound (CUS) is the gold standard to detect pleural adhesions before pleural maneuvers. However, the CUS technique is not available in all countries where the assessment is only based on clinical examination and chest radiography. Objective: To assess the value of lateral decubitus chest radiography (LDCR) to detect pleural adhesions. Methods: Consecutive patients with pleural effusions undergoing LCDR followed by medical thoracoscopy the day after were identified from an institutional database. The diagnostic sensitivity, specificity, positive predictive value (PPV), negative predictive value (NPV), and accuracy for LDCR were calculated. Results: Eighty-six patients were included in the study. The sensitivity, specificity, PPV, and NPV of LDCR for the presence of adhesions taking into account the shape of the horizontal level were $71.2 \%$ (56.7-82.5), 44.1\% (27.6$61.9), 66.1 \%(52.1-77.8)$, and 50\% (31.7-68.3), respectively.
\end{abstract}

The accuracy to predict pleural adhesions for the sign "incomplete horizontal level" was 60.5 (49.3-70.7). The accuracy to predict pleural adhesions in case of irregular aspect of the horizontal level was 53.5 (42.5-64.2). Conclusions: The accuracy of $L D C R$ for the detection of pleural adhesions is low in patients with pleural effusion and LDCR is not sufficient before pleural maneuvers. This has to be taken into account in countries with a high prevalence of pleural tuberculosis which usually lead to loculated pleural effusions. CUS has to be urgently included in dedicated educational programs in these areas in order to decrease the complications related to unexpected pleural adhesions and achieve better planning for the management of pleural effusions.

(c) 2018 S. Karger AG, Basel

\section{Introduction}

During the last 10 years, interventional pulmonology has become more widespread in particular in the field of the management of pleural diseases, mainly pleural effusions [1]. Diagnostic and therapeutic maneuvers are fre-

\section{KARGER}

(c) 2018 S. Karger AG, Basel

E-Mail karger@karger.com

www.karger.com/res
Philippe Astoul

Department of Thoracic Oncology, Pleural Diseases, and Interventional Pulmonology Hôpital Nord, Chemin des Bourrely

FR-13015 Marseille (France)

E-Mail pastoul@ap-hm.fr 

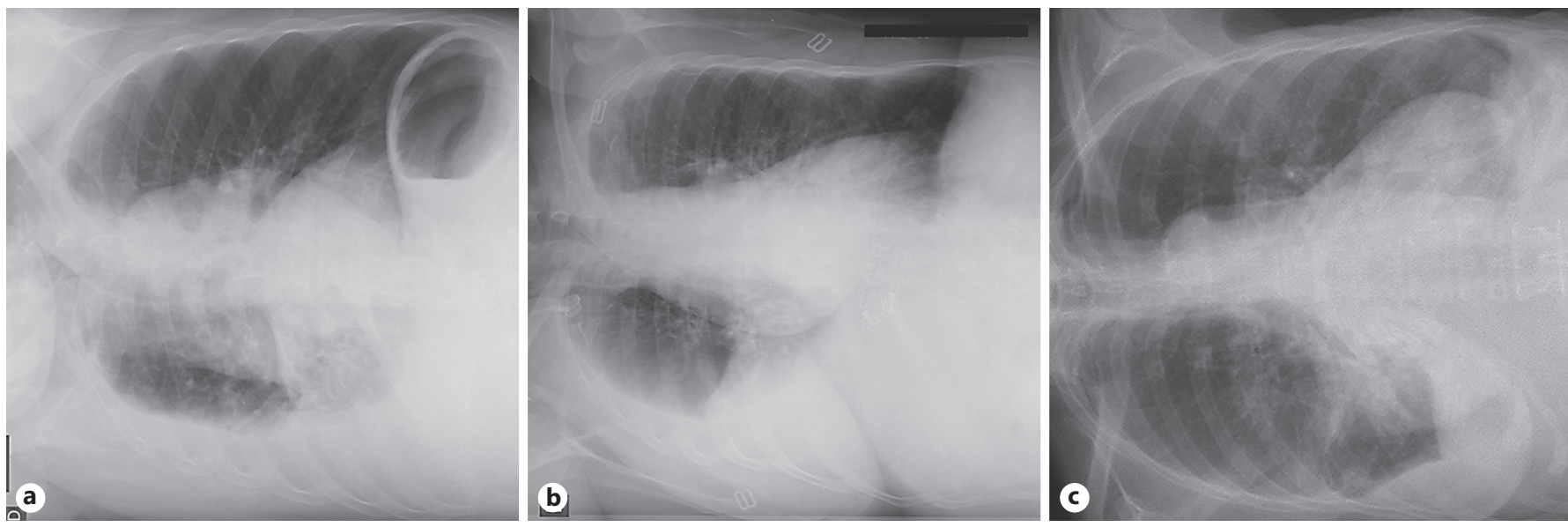

Fig. 1. LDCR evaluations showing complete (a), incomplete (b), and segmented (c) horizontal levels representing the pleural effusion. A complete horizontal level from the diaphragm to the apex was supposed to illustrate free pleural cavity without pleural adhesion.

quently required such as the insertion of pleural drains, thoracentesis, percutaneous pleural biopsy, or medical thoracoscopy. The major drawback is usually the presence of pleural adhesions and their presence is the most common cause of complication during the pleural procedures [2]. The advent of chest ultrasound (CUS) to guide these procedures is clearly a revolution decreasing the complications of such procedures as well as increasing their diagnostic yield [3-5]. This practical approach based on the use of CUS for the management of pleural diseases needs, as a prerequisite, a complete and appropriate training recommended by the majority of the scientific societies [610]. However, even though several training programs are in progress, there are countries where the CUS technique is not available so far and where the pleural approach is only based on clinical examination and chest radiography despite their imprecision $[11,12]$. Because there are few prospective studies on the role of chest radiography before interventional pleural maneuvers [13] and taking into account the lack of CUS in these countries, we tried to assess the value of lateral decubitus chest radiography (LDCR) for the detection of pleural adhesions before diagnostic or therapeutic pleural maneuvers.

\section{Methods}

\section{Study Design and Data Analysis}

From November 2015 to April 2017, all consecutive patients undergoing LCDR followed by medical thoracoscopy the day after for the diagnosis of exudative pleural effusion were identified from our institutional database. All clinical, radiological, and pleural en- doscopy data were also obtained from a prospective institutional database. Data acquisition and analysis were performed after the patients provided informed consent according to local policies and the approval of the local institute review board (Hôpital Nord, Marseille, France). Collected data included demographics (age and gender), macroscopic fluid appearance, volume of fluid, radiography findings on LDCR (complete fluid layer from the diaphragm to the apex, incomplete or segmented fluid layer), thoracoscopy findings, in particular free cavity or presence of adhesions, and final diagnosis after pleural biopsies obtained during the endoscopic procedure.

\section{Pleural Evaluation}

LDCR. Radiographic evaluation was performed using a $125 \mathrm{kV}$ (dose: $25 \mu \mathrm{Gy} / \mathrm{m}^{2}$ ) Optimus 80 unit (Philips) with a $1.5-\mathrm{m}$ filmfocus distance for lateral decubitus views. The exposure was taken in inspiration with the central beam aimed at the lateral chest wall. The radiographic criteria were the thick density with complete (Fig. 1a), incomplete (Fig. 1b), and segmented (Fig. 1c) horizontal level and the limit of opacity, regular or irregular. The complete horizontal level from the diaphragm to the apex was supposed to illustrate free pleural cavity without pleural adhesion. The LDCR analysis was done by two physicians who did not perform the thoracoscopy procedures. In case of disagreement, a third physician was asked for definitive scoring.

Medical Thoracoscopy. Medical thoracoscopy was standardized according to the current European practice as previously described $[14,15]$. The procedure was done under mild sedation or general anesthesia after tracheal intubation with the patient in the lateral decubitus position and spontaneously breathing. An artificial pneumothorax was created on the table before the insertion of a 7 - $\mathrm{mm}$ trocar to allow the introduction of a $0^{\circ}$ telescope. The dedicated intercostal space for the procedure was decided according to the result of preprocedural assessment by chest radiography (erect and lateral decubitus views).

In case of failure to create the artificial pneumothorax, a CUS assessment was performed to detect the potential pleural symphy- 
Table 1. Characteristics of enrolled patients $(n=86)$

\begin{tabular}{lc}
\hline Male, $n(\%)$ & $56(65)$ \\
Female, $n(\%)$ & $30(35)$ \\
Mean age \pm SD, years & $67.86 \pm 14.73$ \\
Fluid appeareance, $n$ & \\
$\quad$ Clear & 36 \\
Bloody & 39 \\
Chylous & 3 \\
ND & 7 \\
Turbid & 1 \\
Mean fluid volume (range), mL & $1,300(20-3,260)$ \\
LDCR findings, $n$ & \\
Complete fluid layer & 25 \\
Incomplete fluid layer & 56 \\
Thoracoscopy findings, $n$ & \\
Free cavity & 34 \\
Adhesions & 52 \\
Final histological diagnosis, $n$ & \\
NSCLC & 66 \\
Mesothelioma & 13 \\
Breast cancer & 3 \\
Gastric carcinoma & 1 \\
B lymphoma & 1 \\
Laryngeal cancer (SCC) & 1 \\
Pachypleuritis & 1 \\
\hline
\end{tabular}

ND, not determined; LDCR, lateral decubitus chest radiography; NSCLC, non-small cell lung cancer; SCC, squamous cell carcinoma.

sis (direct attachment of lungs to the parietal pleura) and guide the choice of another site for a point of entry allowing access to the pleural cavity. Finally, a careful examination of the pleural cavity was done before pleural biopsies under visual control focusing on the presence of pleural adhesions. Medical thoracoscopy was used as the gold standard for the detection of pleural adhesions.

\section{Statistical Methods}

The statistical analyses were performed using the PASW Statistics version 17.0.2 software package (SPSS Inc., Chicago, IL, USA) and the statistical software Vassar Stats. A value of $p<0.05$ was considered statistically significant. Comparisons were made using the $\chi^{2}$ test or Fisher exact test (as appropriate) for the categorical variables. The performance of two signs of LDCR (incomplete horizontal level and/or irregular aspect of the upper limit of the thickness) for the detection of pleural adhesions was evaluated using medical thoracoscopy as the gold standard.

\section{Results}

\section{Epidemiological and Clinical Data}

A total of 86 patients were included in the study, with 56 men and 30 women (35\%) and a median age of 70 years (range: 22-89). The patients' characteristics are summarized in Table 1.

Lateral Chest Decubitus Radiography for Malignant Pleural Effusions
Table 2. Assessment of the pleural cavity by LDCR and thoracoscopy (lesion-by-lesion analysis)

\begin{tabular}{lllc}
\hline Adhesions predicted (LDCR) & Total, $n$ & $\begin{array}{l}\text { Adhesions seen } \\
\text { (thoracoscopy), } n\end{array}$ \\
\cline { 2 - 4 } & & yes & no \\
\hline Yes (incomplete horizontal level) & 56 & 37 & 19 \\
No (complete horizontal level) & 30 & 15 & 15 \\
\hline Total & 86 & 52 & 34 \\
\hline
\end{tabular}

LDCR, lateral decubitus chest radiography.

\section{X-Ray Data}

In 81 patients, the LDCR showed a measurable thick density with horizontal level, indicating the presence of pleural fluid. In these patients, complete and incomplete (i.e., partial) horizontal levels as previously defined were found for 25 and 56 patients, respectively. In 46 patients, the upper part of the thick density was irregular and segmented, which we considered as multilocular pleural effusions. The other 5 patients in whom pleural effusion was not detected were evaluated by ultrasound.

\section{Medical Thoracoscopy Data}

Medical thoracoscopy was performed in 79 patients with pleural effusion and in 7 patients with hydro-pneumothorax. Thoracoscopy assessment of the pleural cavity showed no adhesion in 34 patients, limited pleural adhesions in 33 patients, and diffuse pleural adhesions in 19 patients. These results are collected in Table 2. In case of a complete horizontal level $(n=25)$ or nonvisible $(n=5)$ on LDCR, $50 \%$ of patients (15/30) presented pleural adhesions and 66\% (37/56) in case of partial horizontal level after thoracoscopy assessment. Based on these results, the sensitivity, specificity, positive predictive value (PPV), and negative predictive value (NPV) of LDCR for the presence of adhesions taking into account the shape of the horizontal level were $71.2 \%$ (56.7-82.5), 44.1\% (27.6-61.9), 66.1\% (52.1-77.8), and 50\% (31.7-68.3), respectively. The accuracy to predict pleural adhesions for the sign "incomplete horizontal level" was 60.5 (49.3-70.7) (37 patients with incomplete horizontal sign and adhesions on thoracoscopy, 15 patients with complete horizontal sign without adhesion after pleural procedure). When the upper limit of the thickness on LDCR was regular $(n=35)$ or unseen $(n=5)$, pleural adhesions were found in $57.5 \%$ (23/40) of the cases in the thoracoscopy procedure. In case of an irregular aspect, pleural adherences were found in $63 \%(29 / 46)$ of the cases $(p=0.662)$. 


\section{Discussion}

The purpose of this study was to analyze whether an ipsilateral chest decubitus radiograph correlates with the thoracoscopy findings in patients with pleural effusions focusing on the presence of pleural adhesions. The potential presence of pleural adhesions is crucial to evaluate before all diagnostic or therapeutic pleural maneuvers because of the increasing risk of lung injury and sometimes lack of access to the pleural cavity [16-18]. The prediction of pleural adhesions is useful to choose the port site before a thoracentesis, a percutaneous pleural biopsy or a thoracoscopy [19]. A computed tomography scan has a limited accuracy with a reported sensitivity and specificity of 72 and $71 \%$, respectively [20]. CUS has recently become the gold standard to check the pleural cavity [5]. However, for several reasons, this simple and safe procedure is not available in all countries including those with a high prevalence of pleural diseases. Moreover, it depends on the operator if CUS adds significant value in the identification of pleural fluids and in the pleural procedure guidance. Beside the use of a CT scan, which is also not easily available in some countries, the chest radiograph still remains the initial investigation of choice in patients with pleural disease. It is easy to perform and usually the first diagnostic step in a patient with pleural effusion. However, there are few prospective studies assessing the diagnostic efficacy of chest radiograph and, in particular, LDCR to detect the presence of pleural adhesion in patients with undiagnosed pleural effusion. To the best of our knowledge, this is the first study assessing the role of LDCR for the diagnosis of pleural adhesions using medical thoracoscopy as the gold standard to confirm such a pleural feature. Despite the retrospective analysis of this cohort, this study enrolled consecutive patients undergoing LDCR followed by medical thoracoscopy, if indicated, the day after for the diagnosis of a pleural exudate. According to our results, the accuracy of LDCR for the detection of pleural adhesions in the entire cohort was low whatever the shape of the upper part of the horizontal level. The hypothesis assuming that an incomplete and/ or an irregular aspect of the horizontal level was suggestive of pleural adhesions was not verified and LDCR failed in this intent-to-diagnose pleural adhesion study. This study has several limitations in addition to being retrospective. First, the number of patients was small and a selection bias may have occurred because only patients with an indication of medical thoracoscopy and fitting this procedure were included. However, the prediction of pleural adhesions before medical thoracoscopy is especially mandatory as previously stressed in the literature $[21,22]$. Second, this study did not take into account the number of pleural procedures, mainly thoracenteses, previously done for each patient. Such previous pleural procedures can lead to additional pleural adhesions through the pleural inflammation induced. Third, CUS was performed only in case of suspicion of pleural adhesion and not in the entire cohort. A direct comparison between LDCR and CUS for the detection of pleural adhesion would be of high interest. However, despite these drawbacks, our study may have significant clinical implications showing that chest radiography for the evaluation of pleural effusions is not sufficient before pleural maneuvers for the detection of pleural adhesions. In particular, in several countries with a high prevalence of pleural tuberculosis which usually lead to loculated pleural effusions, other procedures, in particular CUS, have to be spread in order to safely manage such clinical situations. A dedicated educational program in this field is urgently mandatory to decrease the complications related to unexpected pleural adhesions and achieve better planning for the management of pleural effusions.

\section{Financial Disclosure and Conflicts of Interest}

The authors have no conflicts of interest to declare.

\section{References}

\footnotetext{
1 Roberts ME, Neville E, Berrisford RG, et al; BTS Pleural Disease Guideline Group: Management of a malignant pleural effusion: British Thoracic Society Pleural Disease Guideline 2010. Thorax 2010;65(suppl 2):ii32-ii40.

\2 Ferreiro L, Suárez-Antelo J, Valdés L: Pleural procedures in the management of malignant effusions. Ann Thorac Med 2017;12:3-10.
}

\footnotetext{
3 Medford AR, Agrawal S, Bennett JA, et al: Thoracic ultrasound prior to medical thoracoscopy improves pleural access and predicts fibrous septation. Respirology 2010;15:804808.

$\checkmark 4$ Koegelenberg CF, Irusen EM, von GrooteBidlingmaier $\mathrm{F}$, et al: The utility of ultrasound-guided thoracentesis and pleural biopsy in undiagnosed pleural exudates. Thorax 2015;70:995-997.
}

Thiam/Berbis/Laroumagne/Guinde/ Chollet/Dutau/Touré/Astoul 
5 Corcoran JP, Tazi-Mezalek R, Maldonado F, Yarmus LB, Annema JT, Koegelenberg CFN, St Noble V, Rahman NM: State of the art thoracic ultrasound: intervention and therapeutics. Thorax 2017;72:840-849.

6 Board of the Faculty of Clinical Radiology: Ultrasound training recommendations for medical and surgical specialties. 2012. http://www. rcr.ac.uk/docs/radiology/pdf/ BFCR(12)17_ ultrasound_training.pdf.

7 European Federation of Societies for Ultrasound in Medicine and Biology: Minimum training recommendations for the practice of medical ultrasound 2007. http://www.efsumb.org/uploads/mintraining-Feb2006.pdf (February 10, 2012).

-8 Patel SG, Benninger B, Mirjalili SA: Integrating ultrasound into modern medical curricula. Clin Anat 2017;30:452-460.

$\checkmark 9$ Williamson JP, Twaddell SH, Lee YC, et al: Thoracic ultrasound recognition of competence: a position paper of the Thoracic Society of Australia and New Zealand. Respirology 2017;22:405-408.
10 Skaarup SH, Laursen CB, Bjerrum AS, Hilberg O: Objective and structured assessment of lung ultrasound competence. A Multispecialty Delphi consensus and construct validity study. Ann Am Thorac Soc 2017;14:555-560.

11 Eibenberger KL, Dock WI, Ammann ME, Dorffner R, Hörmann MF, Grabenwöger F: Quantification of pleural effusions: sonography versus radiography. Radiology 1994;191: 681-684.

12 Metersky ML: Is the lateral decubitus radiograph necessary for the management of a parapneumonic pleural effusion? Chest 2003; 124:1129-1132.

13 Hallifax RJ, Talwar A, Wrightson JM, Edey A, Gleeson FV: State-of-the-art: radiological investigation of pleural disease. Respir Med 2017;124:88-99.

14 Rodriguez-Panadero F, Janssen JP, Astoul P. Thoracoscopy: general overview and place in the diagnosis and management of pleural effusion. Eur Respir J 2006;28:409-422.

15 Skalski JH, Astoul PJ, Maldonado F: Medical thoracoscopy. Semin Respir Crit Care Med 2014;35:732-743.

16 Seneff MG, Corwin RW, Gold LH, et al: Complications associated with thoracocentesis. Chest 1986;90:97-100.
17 Duncan DR, Morgenthaler TI, Ryu JH, et al: Reducing iatrogenic risk in thoracentesis: establishing best practice via experiential training in a zero-risk environment. Chest 2009; 135:1315-1320.

18 Psallidas I, Helm EJ, Maskell NA, et al: Iatrogenic injury to the intercostal artery: aetiology, diagnosis and therapeutic intervention. Thorax 2015;70:802-804.

19 Diacon AH, Brutsche MH, Solèr M: Accuracy of pleural puncture sites: a prospective comparison of clinical examination with ultrasound. Chest 2003;123:436-441.

20 Mason AC, Miller BH, Krasna MJ, White CS: Accuracy of CT for the detection of pleural adhesions: correlation with video-assisted thoracoscopic surgery. Chest 1999;115:423427.

21 Corcoran JP, Psallidas I, Hallifax RJ, Talwar A, Sykes A, Rahman NM: Ultrasound-guided pneumothorax induction prior to local anaesthetic thoracoscopy. Thorax 2015;70:906908 .

22 Bhatnagar R, Corcoran JP, Maldonado F, Feller-Kopman D, Janssen J, Astoul P, Rahman NM: Advanced medical interventions in pleural disease. Eur Respir Rev 2016;25:199213 